\title{
Émergence de morphologies pour l'acoustique des espaces de performance
}

\author{
Antoine Gros ${ }^{1}$ \\ ${ }^{1}$ MAP-ARIA, UMR CNRS-MCC 3495, 3 Rue Maurice Audin, 69120 Vaulx-en-Velin
}

\begin{abstract}
Résumé. L'acoustique est un vecteur important de qualité de l'ambiance architecturale dans un espace. Elle est aussi une contrainte forte pour le volume correspondant, dont la maîtrise des altérations pour améliorer la perception sonore n'est pas triviale. Nous proposons un outil d'aide à la décision a priori de la forme architecturale, utilisant un algorithme génétique multicritère couplé à un système de simulation acoustique et de calcul des mesures ISO. Son utilisation permet au concepteur l'émersion de morphologies innovantes et qualitatives, aptes à mettre en espace les visions contemporaines des espaces de performance.
\end{abstract}

Mots-clés. Aide à la décision, ambiance sonore, acoustique analytique, algorithme génétique multicritère.

\begin{abstract}
Acoustics is an important vector of quality for the architectural ambiance presented in a space. It is also a strong constraint to the corresponding volume, whose control of alterations to improve sound perception is not trivial. We propose an a priori decision support tool for architectural form, using a multi-criteria genetic algorithm coupled to an acoustic simulation and ISO measurement calculation system. Its use allows the designer the emergence of innovative and qualitative morphologies, able to put into space contemporary visions of performance spaces.
\end{abstract}

Keywords. Decision-making tool, multi-criteria genetic algorithm, architectural ambiance, analytical acoustics.

\section{Introduction}

En dépit du primat de la perception visuelle sur l'ensemble des sens dans les processus de conception architecturale (Pallasmaa, 2010), il est des espaces tels ceux de spectacle et de performance qui polarisent un intérêt évident pour leur qualité acoustique. La bonne perception des sons et la qualité de l'ambiance sonore y sont des exigences programmatiques.

\footnotetext{
${ }^{1}$ Corresponding author: ant.gros@gmail.com
} 
$\mathrm{Au}$ vu de la contingence forte de la forme architecturale sur la conception d'ambiances sonores (Barron, 2009), il est nécessaire d'intégrer les exigences acoustiques au plus tôt dans le processus de conception d'espaces. Son intégration se fait principalement a posteriori de la forme, déjà instanciée en une maquette physique ou numérique (Rindel, 2002 ; 2011) permettant d'évaluer l'acoustique puis d'altérer la forme pour améliorer la qualité d'écoute.

Un nombre limité de formes et volumes sont aujourd'hui identifiés comme fonctionnels et de qualité, ils se perpétuent tout du long de l'histoire de l'acoustique (Beranek, 2003), s'affinent et se maîtrisent au rythme des avancées de cette science succédant ainsi aux démarches pleinement empiriques (Collectif et Mercier, 2002). La réutilisation d'une de ces morphologies apporte une sécurité relative lors de la construction d'un tel espace, l'exemple en est la quantité de salles de concert de type shoebox2 qui sont répertoriées (Beranek, 2003).

La maîtrise des ambiances architecturales est à la croisée de disciplines, l'enjeu est la capacité du concepteur à proposer une expérience $a d$ hoc à un individu dans un milieu conçu. Soit, dans le cas des espaces de performance, de lui permettre d'assister à l'événement dans des conditions de visibilité et d'audition optimales en fonction de sa position dans la salle.

Nous proposons une aide à la conception a priori de la forme architecturale afin d'accompagner l'émergence de nouvelles morphologies pour les espaces de performance. Elles peuvent ainsi être au plus proche des visions, des demandes, des usages contemporains et futurs. Elle consiste en l'optimisation d'une morphologie paramétrée décrite par le concepteur au moyen d'un algorithme génétique multicritère dont la fonction d'évaluation dépend des optimums sur les critères $I S O$ de quantification d'une ambiance sonore (BSI, 2009). Notre étude concernera l'acoustique des lieux de performance pour deux types de sources : la voix, son intelligibilité et la musique, l'estimation de sa qualité.

L'utilisation d'un algorithme génétique multicritère est une solution commune et identifiée pour la résolution des problématiques complexes et interdisciplinaires que suggère la pratique de l'architecture (voir section 3.1.). Cette méthode d'optimisation fournit un corpus d'optimums qui sont autant de suggestions de morphologies adéquates aux demandes du concepteur. De plus, la perception du son étant un phénomène multidimensionnel, il est adéquat d'y faire correspondre une optimisation multicritère.

De tels algorithmes sont mis en place comme aide à la conception et assistance à la prise de décision architecturale et créative (Marin et al., 2009) ; aux problématiques thermiques et d'ensoleillement que ce soit pour l'éco-conception en phase amont (Marsault et Lequay, 2016) ou la conception orientée performance (Turrin et al., 2011) ; à l'optimisation de structure acier (Preisinger, 2013).

Dans le cas de l'acoustique architecturale des salles de concert, l'optimisation d'une forme par un algorithme génétique monocritère a été effectuée en usant de la théorie de la préférence subjective en maximisant un indice composé de la somme des 4 facteurs orthogonaux (Sato et al., 2004 ; Ando, 2009) et par la maximisation de la puissance sonore (Palma et al., 2014). Un algorithme génétique multicritère a été utilisé pour l'optimisation d'une shoebox selon les indices acoustiques $G, E D T$ et $C 80^{3}$ estimés d'après simulation, sans considération pour les critères de spatialité (Echenagucia et al., 2014). Nous nous situons dans la continuité de cette étude par l'utilisation classique du mode opératoire proposé par Barron pour l'emploi de la norme ISO3382-1 (Barron, 2005).

\footnotetext{
${ }^{2}$ Salles rectangulaires comme le Vienna Musikverein en Autriche ou le Berlin Konzerthaus en Allemagne.

${ }^{3} G$, la puissance sonore ; $E D T$, le temps de réverbération du son et $C 80$ l'indice de clarté.
} 
À l'échelle du détail pour l'acoustique architecturale, il faut noter les multiples utilisations de solutions algorithmiques pour l'optimisation de panneaux acoustiques diffuseurs ou absorbants (Rycke et al., 2017 ; Studio, 2013).

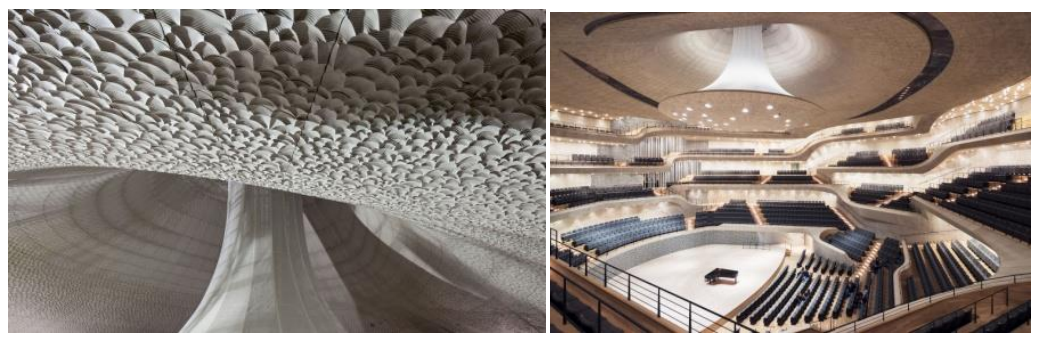

Figure 1. Diffuseurs acoustiques optimisés par algorithme génétique, pour l'Elbphilarmonie, Herzog \& De Meuron.

Pour modéliser la salle en 3D nous utilisons le logiciel de CAO Rhinocéros, le programme est écrit dans son environnement de programmation visuelle Grasshopper. Le plugin Pachyderm (vd Harten, 2013) calcule la réponse impulsionnelle en fréquence, la décroissance d'une impulsion sonore en fonction du temps, en modélisant la propagation du son par diffusion de rayons. Les itérations de l'algorithme génétique sont calculées via le plugin Octopus. L'utilisation de Grasshopper est un choix effectué au détriment de la vitesse de calcul, au profit d'une proximité avec les utilisateurs.

\section{2. Évaluer l'acoustique d'un espace par la mesure}

La branche analytique de l'acoustique architecturale est une science au développement tardif dont l'objectif est de faire le pont entre la perception sonore d'un espace et sa quantification en un ensemble d'indices (Addis, 2009). Le premier critère quantitatif défini par Wallace C. Sabine est le Temps de Réverbération $T R$, comme durée nécessaire à la décroissance d'un son dans l'espace.

La mesure des indices acoustiques des espaces de performance est standardisée par la norme ISO 3382-1. Le TR y est reconnu comme fondamental mais insuffisant à la description de l'ambiance sonore; pour pallier à cela l'annexe I de la norme ajoute 7 critères.

La propagation de la voix et celle de la musique sont similaires, les sons émis concernent les mêmes bandes de fréquence. Des descripteurs analogues sont utilisés pour définir ces sources, la plupart basés sur le rapport entre le carré de la pression sonore reçue sur deux intervalles définis arbitrairement. Nous déclinerons notre approche en deux temps, selon la source, en suivant les recommandations ISO complétées par celles de (Barron, 2009) pour le choix des descripteurs et des objectifs correspondants.

Pour chaque critère, l'objectif est soit une valeur maximale, soit un intervalle. Dans le cas d'un intervalle, nous minimisons l'écart entre la valeur de l'indice et celle de l'intervalle-objectif pour tout récepteur.

Les indices sont estimés sur les bandes d'octaves normalisées entre $62,5 \mathrm{~Hz}$ et $8 \mathrm{kHz}$. Nous avons ajouté le calcul des critères de spatialité $A S W$ et $L E V$ depuis les formules $I S O$ selon l'approximation de $I A C C_{\text {late,mid }}$ par $L F_{\text {late }}$ proposée par (Beranek, 2003), à la bibliothèque Pachyderm par un nouveau module que nous avons codé en $C \#$.

La scène est modélisée sous Rhinocéros où sont disposés source et récepteurs, morphologie paramétrée et statique; à chaque surface sont attachées les caractéristiques acoustiques du matériau envisagé. 


\subsection{Indices acoustiques pour l'intelligibilité des voix}

Outre la puissance sonore, il faut privilégier le son direct et ses premières réflexions pour un temps de réverbération avoisinant $1 \mathrm{~s}$, suffisant pour une bonne intelligibilité et un son " vivant ». La définition - ou Deutlichkeit - est le rapport du carré de la pression sonore reçue $50 \mathrm{~ms}$ après l'arrivée du son direct à la pression totale reçue. Le critère $S T I$ quantifie l'intelligibilité selon le bruit ambiant, c'est un critère normalisé sur l'intervalle $[0 ; 1]$ avec 1 l'indice d'une communication parfaitement intelligible.

Tableau 1. Indices acoustiques optimaux pour une parfaite intelligibilité de la voix.

\begin{tabular}{|l|l|l|}
\hline Nature & Quantité & Objectif \\
\hline Puissance sonore & Loudness, $\mathrm{G}$ & $\mathrm{G}_{\min }>0 \mathrm{~dB}$ \\
\hline Réverbération & Temps de réverbération, TR & $0,8 \mathrm{~s}<\mathrm{TR}<1,2 \mathrm{~s}$ \\
\hline Clarté & Définition, $\mathrm{D}_{50}$ & $\max \left(\mathrm{D}_{50}\right)$ \\
\hline Intelligibilité & $\begin{array}{l}\text { Speech Transmission Index, } \\
\text { STI }\end{array}$ & $\begin{array}{l}\mathrm{STI}>0.8 \text { pour NR25 } \\
\text { bonne, excellente }\end{array}$ \\
\hline
\end{tabular}

\subsection{Indices acoustiques pour la perception musicale}

Les qualités acoustiques attendues d'une salle de concert dépendent du type de musique interprétée. Nous suivrons les recommandations faites pour les grandes salles de concert et d'opéra, à destination de musiques symphoniques. Le $T R$ est plus élevé, la clarté $C 80$ est le rapport de la pression sonore reçue $80 \mathrm{~ms}$ après l'arrivée directe du son à la pression reçue ultérieurement. Le Listener Envelopment LEV indique si le son parait focalisé ou non.

Tableau 2. Indices acoustiques optimaux pour la perception musicale.

\begin{tabular}{|l|l|l|}
\hline Nature & Quantité & Objectif \\
\hline Puissance sonore & Puissance sonore, G & Gmin $>0 \mathrm{~dB}$ \\
\hline Réverbération & Temps de Réverbération, TR & $1,8 \mathrm{~s}<\mathrm{TR}<2,2 \mathrm{~s}$ \\
\hline Réverbération & Durée de Réverbération Initiale, EDT & $1,8 \mathrm{~s}<\mathrm{EDT}<2,2 \mathrm{~s}$ \\
\hline Clarté & Clarté, C80 & $-5 \mathrm{~dB}<\mathrm{C} 80<5 \mathrm{~dB}$ \\
\hline Spatialité & Listener Envelopment, LEV & $\begin{array}{l}\text { Opt }:-2,6 \mathrm{~dB}<\mathrm{LEV}<2,6 \mathrm{~dB} \\
\text { ISO }:-14 \mathrm{~dB}<\mathrm{LEV}<1 \mathrm{~dB}\end{array}$ \\
\hline
\end{tabular}

\section{Optimisation multi-objectifs de morphologies paramétrées}

\subsection{Algorithme génétique}

Un algorithme génétique est un algorithme d'optimisation dont la stratégie est basée sur l'évolution d'une population de solutions vers un optimum théorique. Le processus itératif qui génère les populations est analogue aux mécanismes de la sélection naturelle, où survivent les individus les plus adaptés à l'environnement de solutions.

C'est un processus itératif, chaque itération contenant une population de solutions où chaque individu se différencie par ses variables, composant son génotype.

Le principe général de fonctionnement se décline en 3 étapes : 1) génération aléatoire de la population initiale ; 2) évaluation et sélection d'individus selon leur fitness (déterminée 
par la fonction d'évaluation, définie en 3.2.) ; 3) mutation et reproduction des individus sélectionnés pour constituer la population de la génération suivante.

\subsection{Optimisation}

Les gènes de chaque individu sont les paramètres de la morphologie à optimiser. La fitness pour chaque critère est la somme des distances entre l'intervalle-objectif et la valeur mesurée à chaque microphone pour les bandes de fréquences définies en annexe I de la norme ISO.

Le solveur multi-objectif utilisé est basé sur les algorithmes Strength Pareto Evolutionnary Algorithm-II (Zitzler et al., 2001) et HypE (Bader et Zitzler, 2011). Ils sont tous deux orientés vers la recherche et l'approximation d'ensembles Pareto-optimaux sur plusieurs critères. Ils conservent les individus générés dans un pool de réserve, où ils se répartissent selon leur fitness et leur rapport de domination vis-à-vis des autres individus selon chaque critère. La sélection se fait sous forme de tournois inter-génération, élites et outsiders mêlés afin de compléter le front de Pareto par une variété d'optimums.

Au terme des générations, les individus sont présentés dans Octopus selon 3 catégories : Pareto, Élite, History au sein d'une représentation de l'espace de solutions dans un repère orthonormé. Les caractéristiques des populations sont exportées sur tableur, nous proposons d'y adjoindre une visualisation en ligne sur la plateforme Design Explorer où chaque solution est présentée par une image, un modèle $3 \mathrm{~d}$ et ses caractéristiques acoustiques moyennes (cf. figure 2).

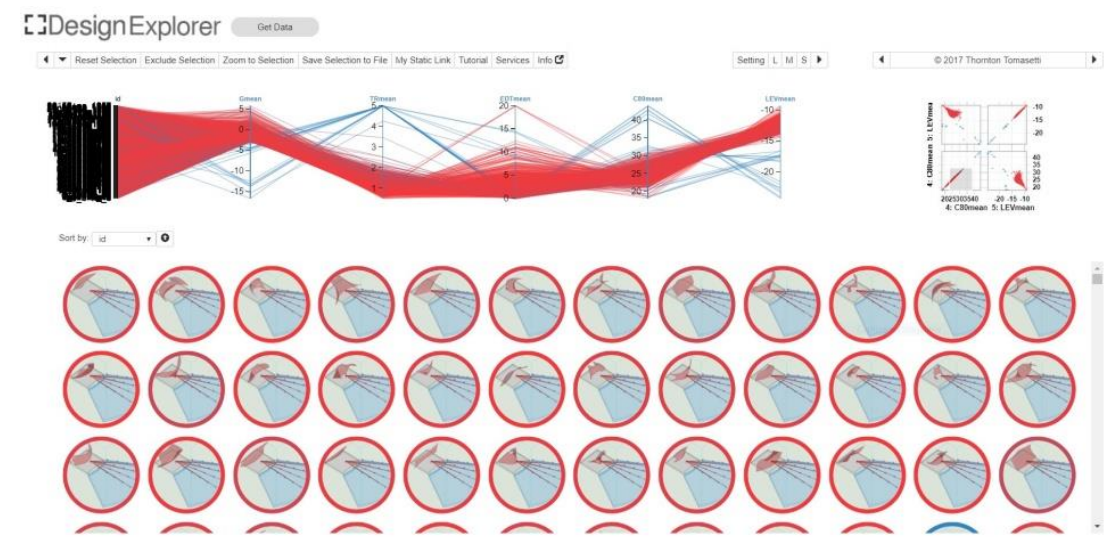

Figure 2. Interface Design Explorer, plateforme de présentation des formes obtenues en fin de processus, ordonnées selon leur performance pour chaque critère. 


\section{Application : Une coque acoustique en béton}

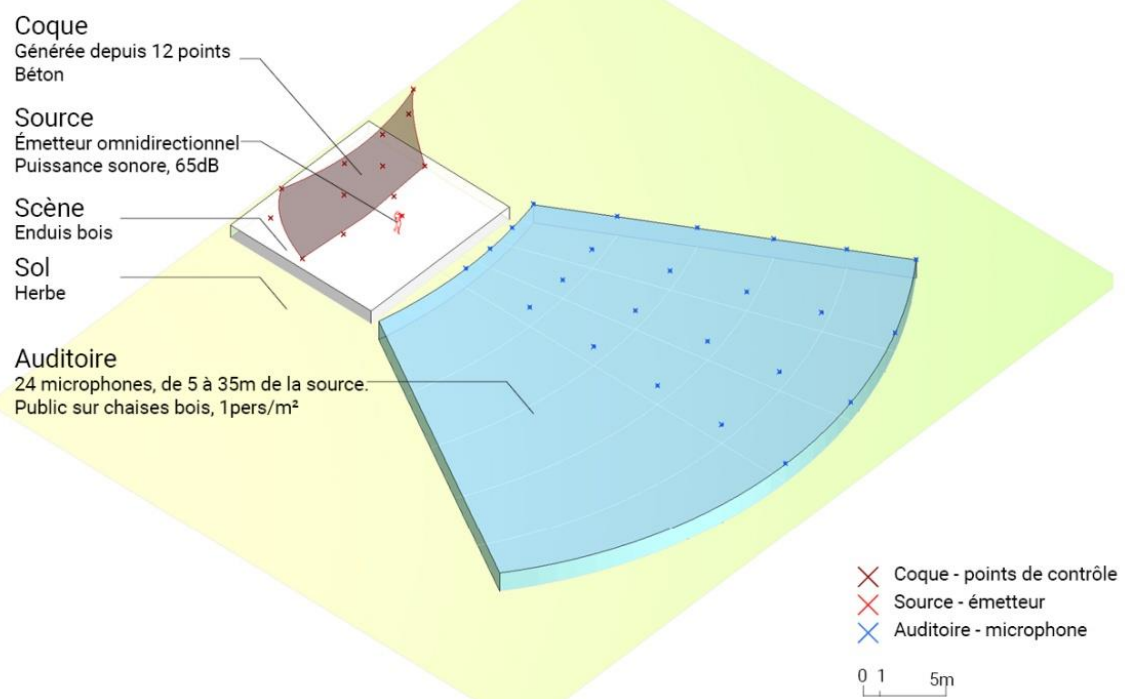

Figure 3. Source et ensemble de microphones.

La forme projetée est une coque en béton à double courbure que nous optimiserons pour la voix puis pour la musique (cf. figure 3), nous présenterons 3 alternatives et leurs valeurs moyennées sur l'ensemble des récepteurs, avec pour référence les valeurs des indices acoustiques pour une surface verticale.

La scène se situe en extérieur, les données acoustiques des matériaux sont définies par (Vorländer, 2007). La coque en béton est symétrique, les 24 microphones sont disposés sur une moitié de l'auditoire pour que chacun ait une valeur unique. Un émetteur omnidirectionnel d'une puissance sonore de $65 \mathrm{~dB}$ est placé sur la scène, surélevée de $1 \mathrm{~m}$ par rapport à l'auditoire. A chaque simulation 15000 rayons acoustiques sont lancés depuis cet émetteur. Chaque individu est évalué par une simulation, l'algorithme génétique itère sur 20 générations de 60 individus.

La coque est une surface $N U R B S$ décrite par la position de ses 4 sommets et 8 points de contrôle uniformément répartis. Les gènes sont les positions en $\mathrm{X}$ et $\mathrm{Z}$ de ces points.

\subsection{Optimisation pour la voix}

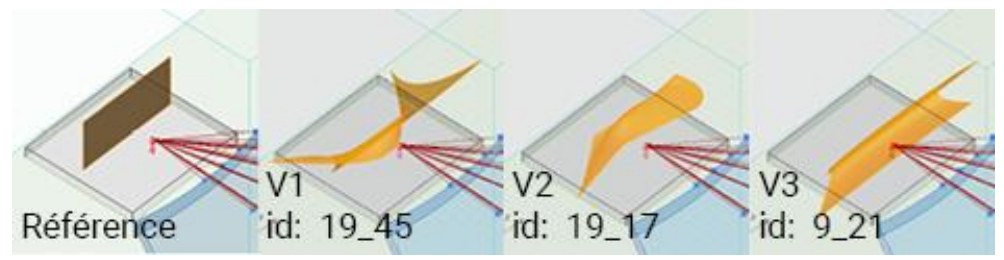

Figure 4. La surface de référence, optimisée pour l'intelligibilité ; l'intégralité des résultats sur Design Explorer, $\underline{\text { https://goo.gl/BtcoMc }}$ 
Tableau 3. Valeurs moyennes des critères acoustiques pour la voix.

\begin{tabular}{|l|l|l|l|l|l|}
\hline$I D$ & Objectif & Référence & $V 1$ & $V 2$ & $V 3$ \\
\hline$G(d B)$ & Gmin $>0 d B$ & -1.33 & 2.90 & 2.85 & 1.02 \\
\hline$T R(s)$ & $0,8 s<T R<1,2 s$ & 1.03 & 0.94 & 0.91 & 0.96 \\
\hline$D 50(d B)$ & $\max (D 50)$ & 99.17 & 99.42 & 99.49 & 99.58 \\
\hline STI $(S . U)$ & $\max (S T I)$ & 0.77 & 0.89 & 0.86 & 0.87 \\
\hline
\end{tabular}

Pour la surface référence, l'indice $G$ indique une propagation de la voix insuffisante. Le temps de réverbération est adapté, mais la voix trop peu puissante est moyennement intelligible $(\mathrm{STI}<0.8)$.

Les trois alternatives présentées sont plus performantes sur tous les critères : la voix se propage mieux, plus clairement, l'indice STI indique une intelligibilité importante.

\subsection{Optimisation pour la musique}

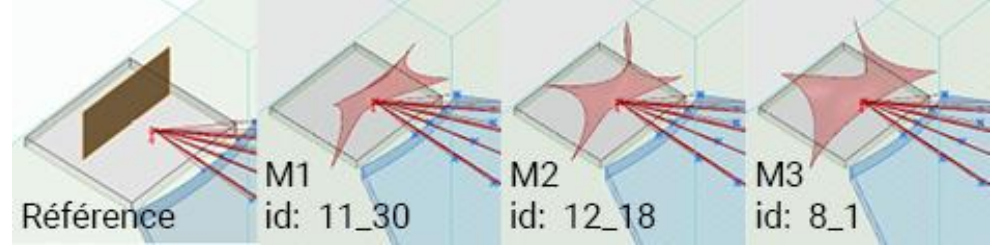

Figure 5. La surface de référence, optimisée pour la perception musicale ; l'intégralité des résultats sur Design Explorer, https://goo.gl/qQJg84

Tableau 4. Valeurs moyennes des critères acoustiques pour la musique.

\begin{tabular}{|l|l|l|l|l|l|}
\hline$I D$ & Objectif & Référence & $M 1$ & $M 2$ & $M 3$ \\
\hline$G(d B)$ & Gmin $>0 d B$ & -1.33 & 5.82 & 1.50 & 0.86 \\
\hline$T R(s)$ & $1,8 s<T R<2,2 s$ & 1.03 & 1.93 & 2.12 & 1.95 \\
\hline$E D T(s)$ & $1,8 s<E D T<2,2 s$ & 4.46 & 0.33 & 0.37 & 0.25 \\
\hline$C 80(d B)$ & $-5 d B<C 80<5 d B$ & 24.36 & 31.20 & 28.32 & 27.32 \\
\hline$L E V(d B)$ & $2,6 d B<L E V<2,6 d B$ & -12.60 & -11.8 & -13.047 & -12.98 \\
\hline
\end{tabular}

Pour la surface référence, l'indice $G$ indique une propagation de la musique insuffisante, le $T R$ est trop bas, le son peu clair et trop dirigé.

Les trois alternatives présentées permettent une propagation de la musique correcte ainsi qu'un $T R$ cohérent aux objectifs. Il y a néanmoins un manque de clarté et un son trop dirigé. Cela est dû à la morphologie envisagée et à l'absence de réflecteurs acoustiques au-dessus ou sur les côtés de l'auditoire. 


\section{Conclusion}

Nous avons présenté une stratégie d'aide à la décision, proposant un ensemble de formes optimales pour une ambiance acoustique prédéterminée, à partir d'une morphologie paramétrée par le concepteur. Nous l'avons illustrée de deux cas d'application, l'une pour l'intelligibilité de la voix et l'autre pour la qualité de l'ambiance musicale.

Les études subséquentes devraient intégrer des notions de propagation sonore dans le solveur afin qu'il soit plus rapide, converge plus rapidement vers les formes selon les critères prédéfinis et évite la production d'individus outliers. D'autre part, il faudrait utiliser un moteur de simulation plus rapide que Pachyderm (Vorländer, 2007).

\section{Bibliographie}

Addis, B., (2009). A brief history of design methods for building acoustics. In : Proc. Third Intern. Conf. Construction History, Cottbus. S.1. : s.n. 2009.

Ando, Y., (2009). Auditory and Visual Sensations. 2010 edition. New York, NY : Springer. ISBN 978-1-4419-0171-2.

Bader, J. et Zitzler, E., (2011). HypE: An algorithm for fast hypervolume-based manyobjective optimization. In : Evolutionary computation. 2011. Vol. 19, n ${ }^{\circ}$ 1, pp. 45-76.

Barron, M., (2009). Auditorium Acoustics and Architectural Design. 2. London: Spon Press. ISBN 978-0-419-24510-0.

Barron, M., (2005). Using the standard on objective measures for concert auditoria, ISO 3382, to give reliable results. In : Acoustical Science and Technology. 2005. Vol. 26, $\mathrm{n}^{\circ} 2$, pp. 162-169.

Beranek, L., (2003). Concert Halls and Opera Houses: Music, Acoustics, and Architecture. 2nd ed. 2004. New York : Springer-Verlag New York Inc. ISBN 978-0-387-95524-7.

BSI, 2009. BS EN ISO 3382-1-2009 Acoustics. Measurement of room acoustic parameters ; Part I Performance spaces. S.1. BSI. ISO 3382.1

Collectif et Mercier, D., (2002). Le livre des techniques du son, tome 1: Notions fondamentales. 3e. Paris : Dunod. ISBN 978-2-10-006304-8.

Echenagucia, T. M., Sassone, M., Astolfi, A., Shtrepi, L. et Van der Harten, A., (2014). EDT, C80 and G driven auditorium design. In : Building Acoustics. 2014. Vol. 21, $\mathrm{n}^{\circ} 1$, p. $43-54$.

Marin, P., Lequay, H. et Bignon, J.-C., (2009). Outil évolutionnaire d'aide à la conception architecturale créative. Mise en oeuvre d'un algorithme génétique et prise en compte des paramètres environnementaux. In : S.1. : CAAD futures. 2009.

Marsault, X. et Lequay, H., (2016). Éco-conception pré-architecturale générative: jusqu'où peut-on aller? In : SCAN'16. 2016. p. 12.

Pallasmaa, J., (2010). Le regard des sens. Paris : Editions du Linteau. ISBN 978-2-91034268-5.

Palma, M., Sarotto, M., Echenagucia, T., Sassone, M. et Astolfi, A., (2014). SoundStrength Driven Parametric Design of an Acoustic Shell in a Free Field Environment. In: Building Acoustics. 1 mars 2014. Vol. 21, p. 31-42. DOI 10.1260/1351010X.21.1.31.

Preisinger, C., (2013). Linking structure and parametric geometry. In : Architectural Design.2013. Vol. 83, $\mathrm{n}^{\circ}$ 2, p. 110-113.

Rindel, J. H., (2002). Modelling in auditorium acoustics. From ripple tank and scale models to computer simulations. In : Revista de Acústica. 2002. Vol. 33, n 3- 4, p. 31-35.

Rindel, J. H., (2011). Room acoustic modelling techniques : A comparison of a scale model and a computer model for a new opera theatre. In : Building Acoustics. 2011. Vol. 18, $\mathrm{n}^{\circ} 3-4$, p. $259-280$. 
de Rycke, K., Gengnagel, C., Baverel, O., Burry, J., Mueller, C., Nguyen, M. M., Rahm, P. et Thomsen, M. R. (éd.) (2017). Humanizing Digital Reality : Design Modelling Symposium Paris 2017. 1st ed. 2018 edition. S.1. : Springer. ISBN 978-981-10-6610-8.

Sato, S., Hayashi, T., Takizawa, A., Tani, A., Kawamura, H. et Ando, Y., (2004). Acoustic design of theatres applying genetic algorithms. In : Journal of temporal design in Architecture and the environment. 2004. Vol. 4, $\mathrm{n}^{\circ}$ 1, p. 41-51.

Studio, LMN Tech, 2013. Acoustic Form Finding with Grasshopper [en ligne]. 4 février 2013. Disponible à l'adresse : https://vimeo.com/58870158.

Turrin, M., Von Buelow, P. et Stouffs, R., (2011). Design explorations of performance driven geometry in architectural design using parametric modeling and genetic algorithms. In : Advanced Engineering Informatics. 2011. Vol. 25, n 4, p. 656-675.

Van der Harten, A., (2013). Pachyderm acoustical simulation : an open source geometrical acoustics laboratory. In : Architectural Design. 2013. Vol. 83, n 2, p. 138 - 139.

Vorländer, M., (2007). Auralization : fundamentals of acoustics, modelling, simulation, lgorithms and acoustic virtual reality. S.1. : Springer Science \& Business Media.

Zitzler, E., Laumanns, M. et Thiele, L., (2001). SPEA2: Improving the strength Pareto evolutionary algorithm. 